\title{
ISOLATION AND CHARACTERIZATION OF A DNA FRAGMENT CONTAINING VARIOUS KINDS OF REPETITIVE SEQUENCES LOCATED ON HUMAN CHROMOSOME 21
}

\author{
Ryoji YAo, ${ }^{1, *}$ David PAtTerson, ${ }^{2}$ and Kazukiyo ONODERA ${ }^{1, * *}$ \\ ${ }^{1}$ Department of Agricultural Chemistry, University of Tokyo, \\ Bunkyo-ku, Tokyo 113, Japan \\ ${ }^{2}$ Eleanor Roosevelt Institute for Cancer Research, \\ 1899 Gaylord St. Denver, CO 80206, USA
}

\begin{abstract}
Summary In order to investigate the repetitive sequences located on human chromosome 21, we have isolated DNA fragments containing Alu sequences. One of the clones, p1, was chosen for further study, because it contained repetitive sequences different from the Alu sequence. $\mathrm{Nu}$ cleotide sequence analysis of $\mathrm{pl}$ indicates that $\mathrm{p} 1$ contains $\mathrm{L} 1$ and $\mathrm{O}$-family sequences. Interestingly, when the $\mathrm{L} 1$ sequence was used as a probe, a discrete band of $5 \mathrm{~kb}$ was seen in HindIII-digested DNA from somatic cell hybrids containing human chromosome 21 as the sole human chromosome. The $\mathrm{L} 1$ sequence was rearranged and was interrupted by O-family sequence, which was flanked by 6 bp target site dup lications. Since all three repetitive sequences are known to act as retroposons, these results imply that there is an integration hot spot on human chromosome 21 . The sequence was mapped within 21q11-21.

Key Words human chromosome 21, repetitive sequence, retroposon, mapping
\end{abstract}

\section{INTRODUCTION}

At least $15 \%$ of the eukaryotic genome is composed of interspersed sequences (Jelinek and Schmid, 1982). The interspersed repetitive sequences have been classified as SINES (short interspersed repeated sequence) or LINES (long interspersed sequence) (Singer and Skowronski, 1985). The major SINES in the human genome

Received February 15, 1993; Revised accepted May 17, 1993.

*Present address: Division of Molecular Genetics, B452, Dana-Farber Cancer Institute, Harvard Medical School, 44 Binney Street, Boston, MA 02115, USA.

**To whom correspondence should be addressed. 
is the Alu sequence family. It has a consensus sequence of about 300 bp and is reiterated 500,000 times in the human genome (Weiner et al., 1986). The major human LINES sequence family is L1 (also described as the KpnI family). The L1 family has a consensus sequence of about $6.4 \mathrm{~kb}$ and is reiterated at least 10,000 times in the human genome. Some of them are internally rearranged or deleted. In addition to these SINES and LINES, retrovirus-like repetitive sequences have been identified. The O-family sequence is one such repetitive sequence and is considered to be a solitary LTR (long terminal repeat) (Sun et al., 1984; Paulson et al., 1985).

Many of these repetitive sequences are transposable elements which can replicate and insert into new positions in the genome to increase their copy numbers. Transposable elements can also facilitate DNA rearrangement such as inversions and deletions (Jagadeeswaran et al., 1982; Lehrman et al., 1986). Sometimes, transposable elements may cause mutations by interrupting genes (Wallace $e t$ al., 1991). Therefore, transposable elements may generate genetic variations in the human genome for natural selection.

Additionally, since a major sequence of human chromosomes consists of repetitive sequences, repetitive sequences may play important roles in chromosome organization. Many single copy DNA fragments have been mapped because of their usefulness for landmarks of each chromosome. However, mapping repetitive sequences in human chromosomes has been difficult precisely because they are reiterated many times, and the distribution of repetitive sequences remains somewhat unclear.

In many mapping efforts, human chromosome 21 has served as a prototype for the human genome. Physical, genetic, and compositional maps of human chromosome 21 have been reported (Petersen et al., 1991; Gardiner et al., 1990a). The distribution of unique sequences, rare restriction enzyme sites, $\mathrm{CpG}$ islands and chromosomal break points has been described (Gardiner et al., 1990b).

In order to investigate further the organization of human chromosome 21, we have isolated DNA fragments containing Alu sequence from mouse-human somatic cell hybrids containing human chromosome 21 as the only human chromosome. Here we report the characterization of one of the clones which contains various kinds of repetitive sequences.

\section{MATERIAL AND METHOD}

Somatic cell hybrids. The human and rodents cell lines, as well as human/ rodent somatic cell hybrids, WA17, 2Fur, ACEM, R2-10, and $21 \mathrm{q}^{\dagger}$ cells were used and their properties have been described previously (Oates and Patterson, 1977; Kozak et al., 1977; Van Keuren et al., 1986; Patterson et al., 1983; Wong et al., 1989; Drabkin et al., 1895).

Southern blot analysis. Chromosomal DNA was isolated according to a method 
described by Zsebo et al. (1990). DNA (10 $\mu \mathrm{g})$ was digested with HindIII, developed on an agarose gel, and blotted onto GeneScreenPlus (New England Nuclear) membranes. These blots were prehybridized in a solution containing $50 \%$ formamide, $5 \times$ Denhardt's solution $(1 \times$ Denhardt's solution $=0.2 \mathrm{~g} / \mathrm{l}$ Ficoll, $0.2 \mathrm{~g} / \mathrm{l}$ polyvinylpyrolidone, $0.2 \mathrm{~g} / \mathrm{l}$ BSA), $0.5 \% \mathrm{SDS}, 100 \mu \mathrm{g} / \mathrm{ml}$ sheared, denatured salmon sperm DNA, $6 \times \mathrm{SSC}(1 \times \mathrm{SSC}=0.15 \mathrm{M} \mathrm{NaCl}, 0.015 \mathrm{~m}$ sodium citrate $)$ at $42^{\circ} \mathrm{C}$ for $2 \mathrm{~h}$, hybridized with ${ }^{32} \mathrm{P}$-labeled probes in a solution containing $50 \%$ formamide, $0.5 \%$ SDS, $100 \mu \mathrm{g} / \mathrm{ml}$ sheared, denatured salmon sperm DNA, $6 \times \mathrm{SSC}$ at $42^{\circ} \mathrm{C}$ for $40 \mathrm{~h}$, and washed at a final stringency of $0.1 \times \mathrm{SSC}, 0.2 \% \mathrm{SDS}$, at $65^{\circ} \mathrm{C}$.

DNA sequencing. Each fragment was subcloned into pUC118 or 119 and nested deletions were generated by progressive digestion with exonuclease III (Henikoff, 1984). Automated analysis of DNA sequence was performed by the chaintermination method, adopted for ssDNA using Taq dye-primer sequencing kit (Applied Biosystems, Foster City, CA) by means of an ABI 370A DNA sequencer (Applied Biosystems). Sequences were analyzed using software provided by the Genetics Computer Group.

Polymerase chain reaction (PCR). PCR was performed essentially as described by Saiki et al. (1988). A set of primers, F2, 5'-CTTAGACATAGATGTCTGCC and R2, 5'-GGTACTTCCATTAAACTACC were synthesized according to the p1 nucleotide sequence at the position betewen 547 and 566 and at the position between 1501 and 1520, respectively. A set of primers which was designed to amplify exon 15 of amyloid protein precursor (APP) was used as internal controls (Levy et al., 1990). The sequences of APP primers are 5'-CCTCATCCAAATGTCCCCGTCATT and 5'-GCCTAATTCTCTCATAGTCTTAATTCCCAC. PCR was carried out in $100 \mu \mathrm{l}$ of reaction mixtures containing PCR buffer $(50 \mathrm{mM} \mathrm{KCl}$, $10 \mathrm{~mm}$ Tris- $\mathrm{HCl}, \mathrm{pH} 8.3,1.5 \mathrm{mM} \mathrm{MgCl}_{2}, 0.01 \%$ gelatin), $20 \mathrm{nmol}$ each dNTP, 100 pmol of each primer, $0.1 \mu \mathrm{g}$ DNA, and 2.5 units of Taq polymerase and was covered with $100 \mu \mathrm{l}$ of mineral oil. The reaction was carried out for 30 cycles. One cycle was denaturation $\left(94^{\circ} \mathrm{C}, 1 \mathrm{~min}\right)$, annealing $\left(58^{\circ} \mathrm{C}, 1 \mathrm{~min}\right)$, and extension $\left(72^{\circ} \mathrm{C}, 2\right.$ $\mathrm{min}$ ). Initial denaturation was done for $5 \mathrm{~min}$ and the final extension was done for $10 \mathrm{~min}$. The PCR products were analyzed on $3 \%$ NuSieve GTG agarose gels (FMC Bioproducts, Rockland, ME).

\section{RESULTS}

\section{Southern blot analysis of clone pl}

In order to isolate genomic sequences encoded by human chromosome 21, the genomic DNA from human-mouse somatic cell hybrid WA17, which contains human chromosome 21 as its only human chromosome, were isolated and cloned into lambda ZAPII vector to construct a genomic library. The genomic library was screened with an Alu sequence, BLUR-8 (Rubin et al., 1980), as a probe by means of plaque hybridization. Positive clones were converted into pBluescript- 
SK $(-)$ and were used for further studies. To characterize further, each clones was subcloned and the subcloned fragments were subjected to the Southern blotting hybridization analysis. On this way to identify the location of Alu sequence, we happened to find that one of the clones, $\mathrm{p}$, contains repetitive sequences in addition to the Alu sequence and that it has a distinctive structure. The restriction map of clone pl is shown in Fig. 1A. Clone pl has a size of approximately $4 \mathrm{~kb}$ and $\mathrm{Alu}$ sequence was found in the indicated XbaI-BglII fragment (Fig. 1A). When the KpnI-Xhol fragment (Fig. 1A, probe C), which does not carry Alu sequence,

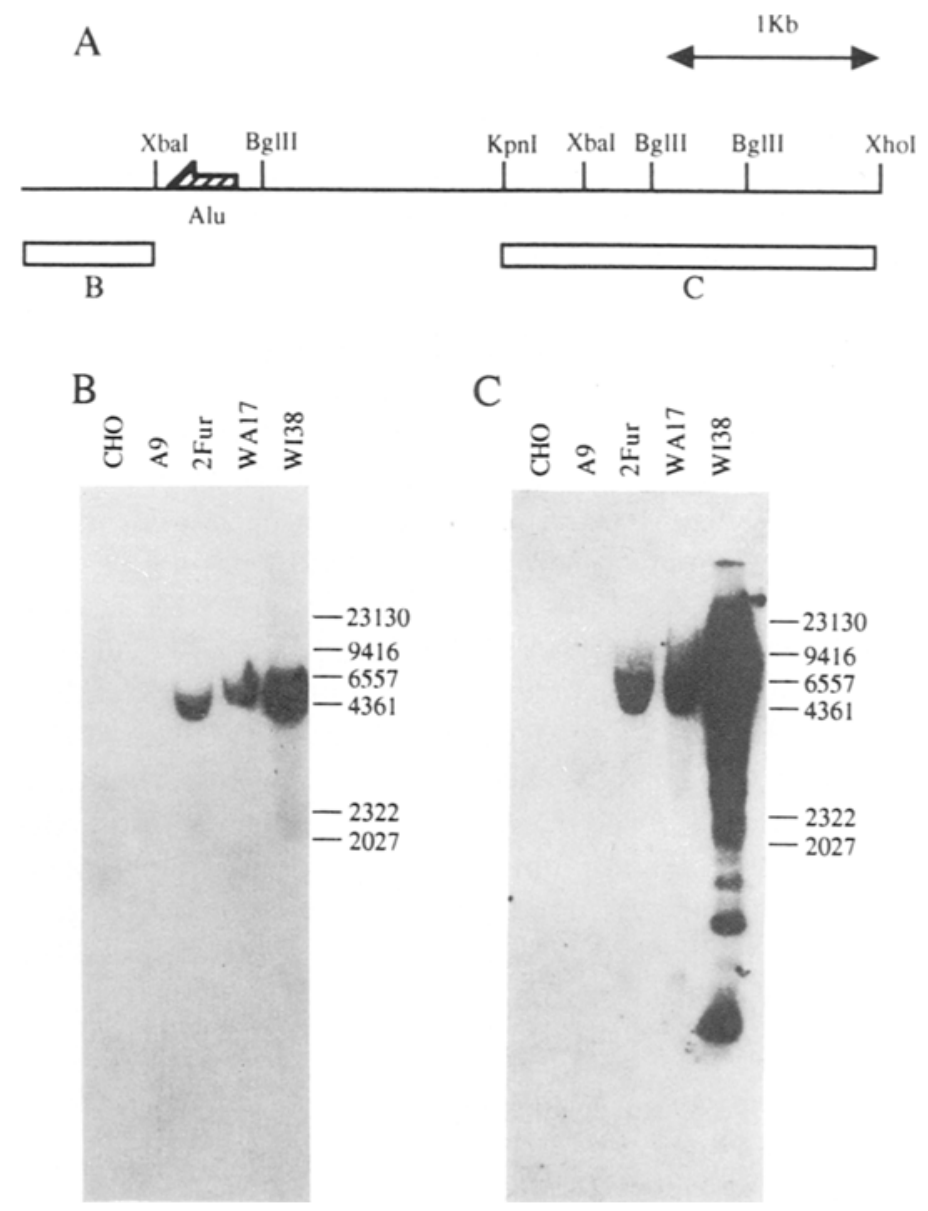

Fig. 1. A: Restriction map of p1. Probe for Southern hybridization are shown as open boxes. An arrow represents the location and orientation of Alu sequence. B: Southern hybridization analysis of clone p1. DNAs from cell lines indicated at the top were digested with HindIII, applied on $0.7 \%$ agarose gel, and transferred to a nylon filter. The filter was hybridized with probe B (panel A). C: A same series of Southern hybridization analysis was carried out using probe C. 
was used as a probe, Southern blot hybridization analysis of HindIII-digested human DNA revealed a smear suggesting that the fragment contains repetitive sequences different from Alu sequence (Fig. 1C).

Interestingly, a discrete band of $5 \mathrm{~kb}$ was seen in HindIII-digested DNA from WA17 or 2Fur cells, which are somatic cell hybrids containing human chromosome 21 or the long arm of human chromosome 21 as their only human chromosome material respectively. The intensity of the band was much stronger than that given by single copy genes (data not shown). These results suggest that the repetitive sequences contained in the K pnI-Xhol fragment are dispersed in human chromosomes in HindIII fragments of different sizes. However, on human chromosome 21, the repetitive sequence appears to exist in HindIII fragments of a single size. These results suggest the possibility of the specific and/or dominant organization of the repetitive sequence on human chromosome 21 .

\section{Sequence of clone $p 1$}

The complete sequence and the organization of the clone $\mathrm{p} 1$ is shown in Figs. 2 and 3. The Alu sequence was found to be located at the position between 608 and 922 and was flanked by $10 \mathrm{bp}$ target site duplications (TSDs). The Alu sequence in clone p1 was $80 \%$ homologous to that of consensus sequence (Jurka and Smith, 1988).

The region homologous to $\mathrm{L} 1$ repetitive sequence was found in between nucleotide position of 1021 and 4142. This region has an unique structure. The first $2640 \mathrm{bp}$ (nucleotide position 1021 to 3660 ) is homologous to L1 sequence (nucleotide position 2269 to 4584 of the L1 sequence) in inverted orientation (Skowronski et al., 1988). On the contrary, the second part (nucleotide position 3668 to 4142) was also homologous to L1 sequence (nucleotide position 1024 to 1502 ) in the forward orientation. The sequence of 7 nucleotides between the two parts was not homologous to the $\mathrm{Ll}$ sequence in either orientation. No open reading frame (ORF) was found in this L1 homologous region.

In addition to Alu and L1 sequences, O-family repetitive sequences (Sun et $a l ., 1984$ ) were found at the position between 1076 and 1418 . The O-family repeat is flanked by $6 \mathrm{bp}$ TSDs. The O-family sequence is believed to be a solitary LTR-

Fig. 2. Nucleotide sequence of clone $\mathrm{p} 1$ and its comparison with repetitive sequences. The p1 sequence is shown on the top and Alu, L1, and O-family sequences are in the second or third lines. Alu indicates the Alu sequence described by Jurka and Smith (1988); O-F, O-family sequence described by Sun et al. (1984); L1-R, L1 sequence described by Skowronski et al. (1988) in reverse orientation; L1, L1 sequence described by Skowronski et al. in forward orientation. Numbers indicate nucleotide positions corresponding the original reports. Dots represent the identical bases and dashes represent deletion. The box indicates the sequence between two regions homologous to the $\mathrm{Ll}$ sequence in different orientation. Heavy underlines show target site duplications (TSDs) for the Alu sequence and light underlines show TDSs for the O-family sequence. 

61:TATGTTTCTG AATGACCAGT GGGTTAATGA ATAAATAAAC AGAAAATTTT AAAACGTTTT 121: TAAGCAAATA ACAATGGaAg CACAACATAT CAAAATGTGT GGCATACAGC AACAGAaGTA 181: CAAAGAGTAA TITTTTHTTA TTATACTTTA AGTITITAGGG TGCAAAGAGT AAATTTTAAG 241: CCTATAAGTG CCTACATCAA AAAGGATAA AAACTTCAAA TTAACTATCT AATGGTGCAT

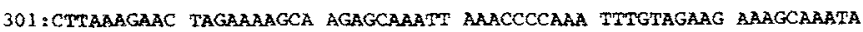
361 : ATAAAGATCA ATCAGAAATA AATGAAATIG AAATGAAAA ACAATACAAA AGGCCAATGC 421: AATAAAATAT TEGTTTTIIG AAAAGATAAA ACTGACAAGC CTTTAGCAGA CAAATTAAGA 981: ACAGAGATGG CCCAAATAAA TAAAGTCAGA GCAAAATACA GAGAATCTAG AATAGATAGA 541: GAAATTCTTA GACATAGATG TCTGCCTACC AAGAGTAAGC CATGAAGAAA IUTGAAGCAT

Pl 101 : 1 AAATAGAGGC TGGGCACGGT GGCTCATGCC TGTAATCCCA GCACTTTTGC GAGACCAAGG

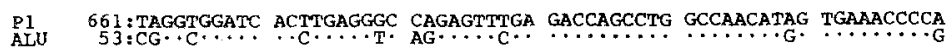
P1 211 :TCTCTAGTAA AAACACACAC AAAAATTTAG ACAAGCATGG TGACATGCAA TMGTAATCCC

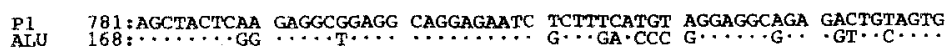
P1 241 : AGCTGAATTT GCACCACTGC ACTCCAGCCT GGGCAACAGA GTAAACTCC ATCTCAAAAA P1 $901:$ ACAAAACAAA ACAAAAAAAA CACATAAATA GACCAATAAC AAATAACAGA TMTAAACCAT P1 961: AAAAGACTGT ATCACAGCAA AGAAAAGCCC TGGGACCTGA TGAATTCTTT GCTGAATTPT

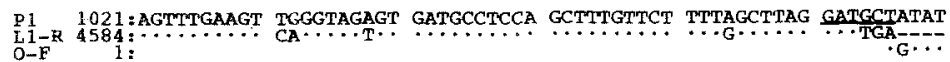

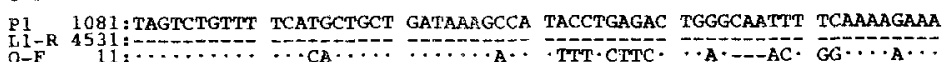
P1 $1141:$ GCAATTTAA- TGGACTTACA ATTCCATOTA GCTGGGGAGG CCTCACAATC ATGGTGGAGG

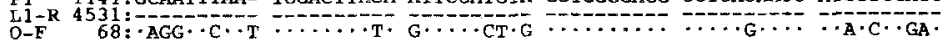
P1 $1200:$ GTGAAAGGCA TGXCTCACAT GGTGGCAGAC AAGAGAAGA GGTTG-TGCA GGGAAA-.--

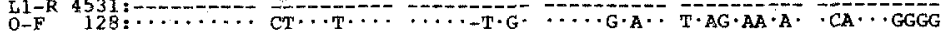
P1 1255 : CACCTCCTTA TATAATTGTC AGATCTCATG AGACTTATTC ACTGTCATGA GAACAGCACA

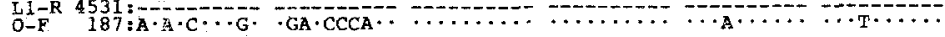
P1 1315 : GGAAAGACCT GCCCCTGTGA TTCAATHACC TCCCACCAGG TCCCTCCCAC AATACGTGGG

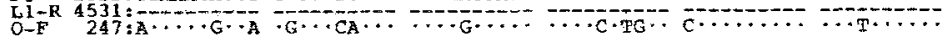
P1 $1375:$ AA- 1 -

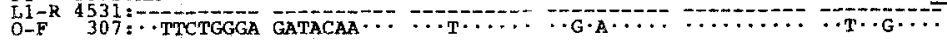
P1 $1420:$ ATGCATMGC TAMTCAGGCT CTCMTTTGGT TCCATATGAA TTMPAAAATA GTTTTTTTCT P1 1480 :AGTTCTGTEA AGACTGTCAA TGGTAGTTTA ATGGAAGTAC CATTGAACGT ATAAATTGCT P1 $1540:$ TIGGATAGTA TGGTCATTT AATGCTATTG ATTCTTCCCA TGCATGAGCA TGGAATGTT

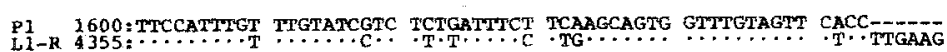

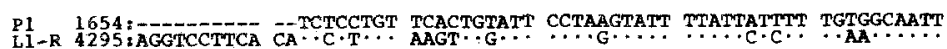

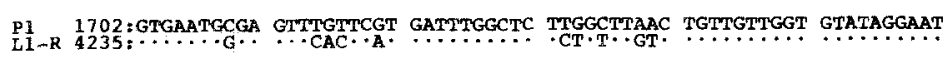
P1 1762:GTTAGTGA-T TPGCACATT GATTMTGTAT CTTGAGATTT TGCTGAAGTT GTTTATCACC P1 $1821:$ TTAAGAGCT TTTGGGCCA GACTACAGGG TTTCTACAT ATAGGATTAT GTCATCTGCA P1 1881:AACAGAAATA ATTTGACTGC CTCTCTTCCT GTTTGACTGC CTTMTATTTC TTTCTCTTG-

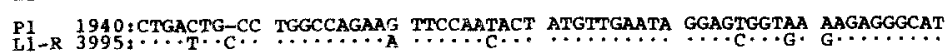
Fig. 2. P1 $1999:$ CCMTGTCTTG TGCTGGCTTT CAAGGGGAT TCTCCAGCA TTTGCCCAGT CAGTATGGTG 
P1 2059:CTGGTTGTGG GTTTGTCACA GATGACTCTC ATPATTTTGA GGTATGATCC ATCACTACCT

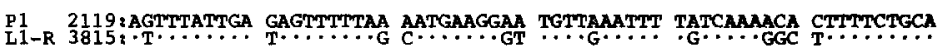
P1 2179 : TCTATTGAGA TAATCATGTG GNTTTTGTCT TAGTTPTTTG CCTPTGTTTA CGTGATGAAT P1 2239: CACATTTATT GATTTGCATA TGATGAACCA ACCTAGCATC TAGGGGATGA ATCCTACTTA P1 2299:CTCATAGTGA ATACACTTTT TGA-R-TG CTGAATTTGG TTTGCCAGTA TTTPTTTG-G P1 2353:ATTTTTTTGAA TTGATGTTCA TCAAGGATAT TGGCCTGAAG TTTPCTTATT TTGTTGTATC

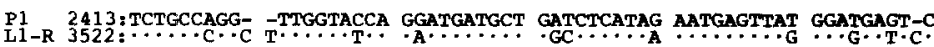
P1 $2470:$ CPCTTCTTCA CTTCITTGGA ATAGTTTCAG TAGAGATGGT ATCAGCTCTT CTPTGCACTT P1 $2530:$ CTGETAAATT TCAGCTGTGA ATCTGTCTTA CCCTGAGCTT TTTAAGGTPA GTAGGCTATT P1 $2590:$ TATTACTACC TCAATTPGAA AACACACTTPT TGGTCTGTTC AGGAATTCCA TTTCTACCTG P1 $2650:$ GTPCAGTATT GGGAAGGTT ATGTGCCCGT GAATTTATTC ATTTCTICTA GATHTTCTAG P1 $2710:$ TTTGTGTCTA TAGAGGTATT TATAATATTC TCCGATGGTP GTTTGTATTT CTGTGGGGTC

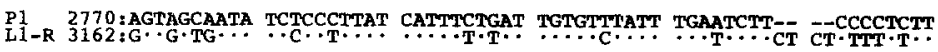

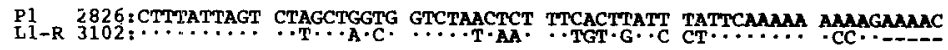
P1 2886:ACCTCCTGAA TTTGTTGGG PTTTGAATGG TCTTPCATGT CTCTATCTCC TCCAGTTCAG

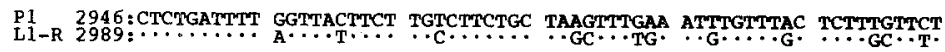

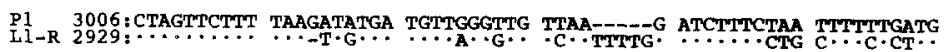
Pl 3061 :TGGGCACTTA ATGCTATAAA TTTPCATCTT AACACTGCCT TAGCTGTGTC CCAGAGATTC Pl $3121:$ TGGTAGGTT TATCTTTTTT TCTCATTAGT TTCAAATAAC TTCTTGCTTT CTGCCTTAAT PI $3181:$ TTCATTATTP TCCCAAACGT TATTCAGGAG CAGGTTATTC AATTTCCATG TAATCATACG

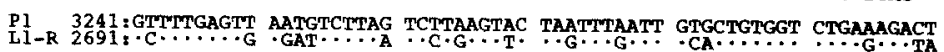

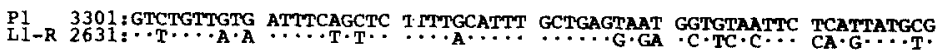
P1 3361:ATPAATTPTA GAGTAAGTAA TATGTGGCAA TGAGAAAAT GTATPTPCAG TTGTTCTGGG P1 3421 : GTGGAGAGTT TGGTAGATAT TTATCAGGTT TATTPTAATC CAMAGCTGA TTCAGGTCCT P1 $3481:$ GAATATCTTT CTPACATTTC TGTCTCAATG A-M-TCTAA TATTGACAGT GGGGTGTTAA P1 $3537:$ ATTCACCCAC TPTTATTGTG T- T-GAGTCTA AGTCTCTCTG AAGA PCTCCA AGAMATTGAT P1 $3595:$ TTACCAATAT GGGTGCTCCT ATGTTGGGTG CATATATATT TAAGATAGTT AGCTCPTCTT

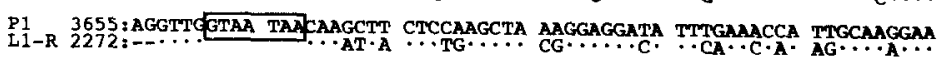
P1 $3715:$ GCTAAAAAC - TTGAAAAAG ATTAGACAAA TGGCTAACTA GAATAAACAG TGTMAAGAG P1 3774 : AACTPAAATG ACCTGACGGA GCTGAAAACC ATGGCACAAC AACTTCGTGA CGCATGCACA P1 $3834:$ AGCATCACTA GCCAATGGG TCAAGAGGAA GAAAGGGTAT CAGTGACTGA AGATCAATT

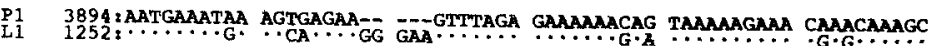
P1 $3949 ;$ CTCCAAGAAA TATGGGACTA TGTGAAAAGA CCAAATCTAC ATTTGATGGG TGTACCTGMA

PI $1009:$ AGTAACAGG AGAATGGAAC CAAGTGGA AACACTCTTG AGGATATTAT CCAGGAGAAC P1 4069 : TTCCCCAACC TAGCAAGGCA GGCAACATT CAMATTCAGG AMATACAGAC MACACCACA

P1 $1129:$ AGATACTCCT CGAG

Vol. 38, No. 3, 1993 
like sequence, since it starts with TG and terminates with CA, which is a hallmark of the LTRs (Varmus, 1982). The O-family sequence located in p1 starts with TA and terminates with CA. Deviation from the "TG-CA" rule applicative to the infectious retroviruses has also been seen in the LTRS of a previously described class of human retrovirus-like sequence (Steele et al., 1984; Mager and Henthorn, 1984). The LTRs described by Mager et al. also start with TA. It is also interesting to note that the O-family sequence in clone pl was found within the $\mathrm{L} 1$ sequence, because both of the retrovirus-like sequences described by Steele et al. and Mager et al. were also found within the L1 sequence. These observations may indicate the association of some particular family of retrovirus-like sequence with Ll sequence.

A region between nucleotides 1 and 608 which did not contain any repetitive sequence was AT-rich and the $\mathrm{GC}$ content of the region was $30 \%$. It has been suggested that retroposons such as Alu sequence are preferentially inserted into short AT-rich regions (Daniels and Deininger, 1985). This AT-rich region of p1 could be preferred for the insertion of such various kinds of repetitive sequences.

\section{Regional assignment of clone pl}

To determine the regional location of clone $\mathrm{p} 1$, the location of $\mathrm{pl}$ sequence in somatic cell hybrids containing various regions of human chromosome 21 was analyzed by PCR. A set of primers designed according to the pl sequence was used. Primers for APP were added to the reaction mixture as internal controls. The amplified fragments are expected to contain both Alu and O-family sequences (Fig. 3). When the DNAs from 2 Fur, WA17, R2-10, and $21 \mathrm{q}^{+}$cells, somatic cell hybrids containing 21q11-21, were used as template, fragments with the expected sizes were amplified (Fig. 4A). The fragment was not amplified either from DNAs of hamster or mouse cells or from somatic cell hybrids, $8 q^{-}$and ACEM. When p1-specific primers were used, only the upper bands were seen (data not shown).

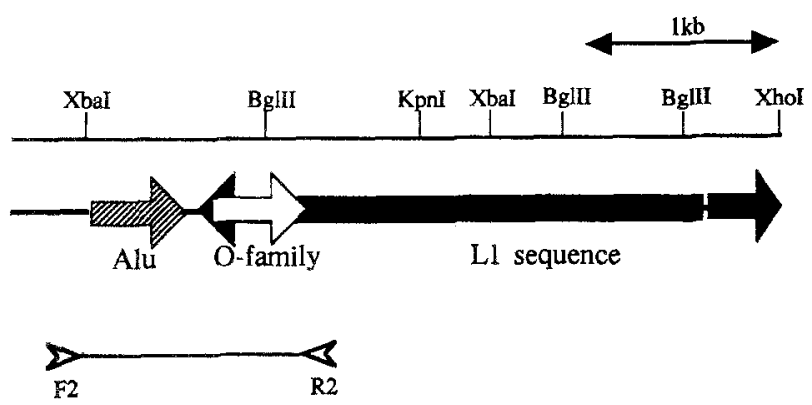

Fig. 3. Schematic diagram of p1. Restriction sites of $p 1$ are shown on the top. Shaded arrow indicates Alu sequence; open arrow, O-family sequence; closed arrow, L1 sequences. The p1 specific primers, F2 and R2, are shown by small open arrows, which are located at both ends of the amplified fragment indicated by thin line. 

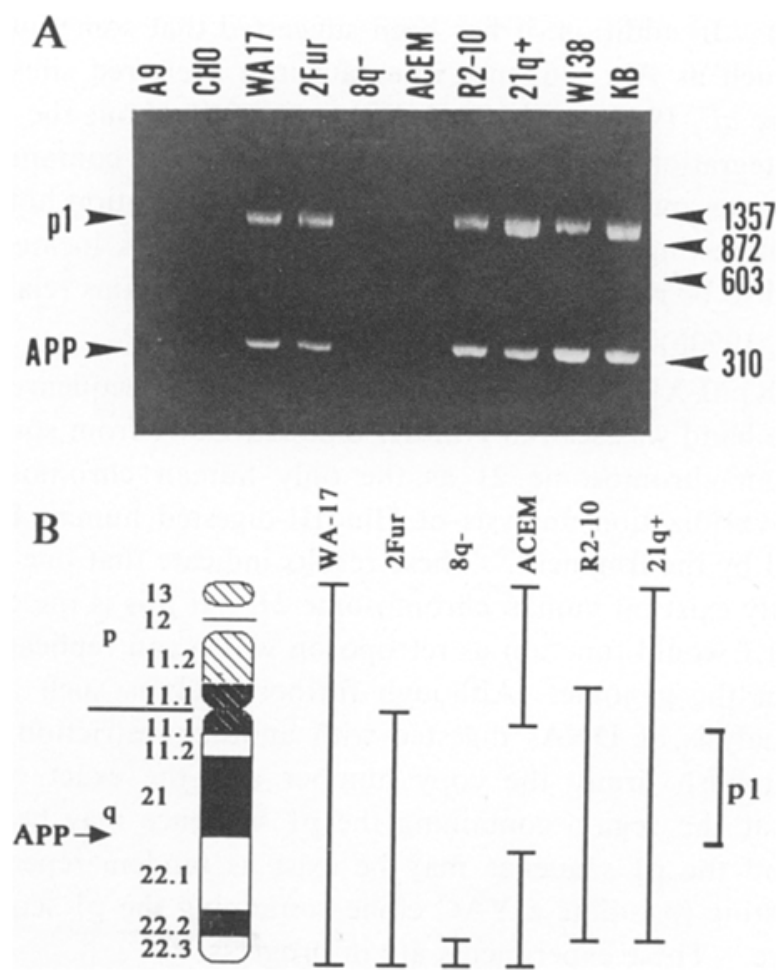

Fig. 4. PCR analysis of human-rodent hybrid cell panel. A: DNAs from cell lines shown on the top of the figure were subjected to PCR analysis with primers specific to $\mathrm{p} 1$ and amyloid protein precursor (APP) sequences. Amplified DNAs were visualized by ethdium bromide staining. p1-specific DNA fragments are indicated on the left. The numbers indicate positions for the size markers. B: Chromosomal segments contained in somatic cell hybrids. Idiogramatic representation of chromosome 21 is shown in the right part. The location of APP is shown by arrow. The region in which clone $\mathrm{p} 1$ was mapped is shown in the right bracket.

These results indicate that $\mathrm{pl}$ is located within 21q11-21 (Fig. 4B). Since fragments with the expected size were amplified, the structure of the p1 sequence may be conserved among each human genome of these cells.

\section{DISCUSSION}

The human genome is composed of two structurally distinctive regions, Gbands (Giemsa positive, or Giemsa dark bands) and R-bands (reverse bands; these are equivalent to Giemsa negative, or Giemsa light bands). It has been suggested that some classes of repetitive sequences are clustered in specific bands, i.e., Alu sequence predominates in the R-bands and $\mathrm{L} 1$ in the G-bands (Korenberg and 
Rykowski, 1988). In addition, it has been suggested that some interspersed repetitive sequences such as Alu sequence integrate into preferred sites in the chromosomes (Moyzis et al., 1989). Although little is known about the molecular mechanism of the integration of retroposons, the fact that pl contains three kinds of putative retroposons may indicate that there is an integration hot spot. If this is the case, it is interesting to note that it is likely that pl is located in the G-band which is believed to be relatively gene poor and which contains relatively few known genes (Gardiner, 1990b).

When the KpnI-Xhol fragment, which contains Ll sequence, was used as a probe, a discrete band was seen in HindIII-digested DNA from somatic cell hybrids containing human chromosome 21 as the only human chromosome. However, Southern blot hybridization analysis of HindIII-digested human DNA resulted in a smear detected by the fragment. These results indicate that one kind of structure would dominantly exist on human chromosome 21 . If this is the case, it is difficult to explain how L1 would function as retroposon which can replicate and insert into new positions in the genome. Although further analysis such as Southern blot hybridization analysis of DNAs digested with another restriction enzymes will be necessary to establish firmly the copy number and the exact organization, one possibility is that the region containing the $\mathrm{p} 1$ sequence may be amplified at the genome level and the pl sequence may be exist as tandem repeat. Therefore, it would be interesting to isolate a YAC clone containing the p1 sequence and determine its structure. These experiments are in progress.

The clone pl contains three kinds of retroposons, Alu, L1, and O-family sequence (Weiner et al., 1986). The function of these retroposons remains unknown. However, there is suggestive evidence that some retroposons may be involved in genome rearrangement (Jagadeeswaran et al., 1982; Lehrman et al., 1986; Henthorn et al., 1986). In particular, the retrovirus-like sequence at the globin locus is supposed to facilitate recombinational events to produce thalassemias (Mager et al., 1985). Alternately, the presence of such repetitive sequences may indicate that the region is particularly susceptible to breakage for the integration of foreign DNA. Precise mapping of $\mathrm{pl}$ and further analysis of this locus may provide further clues to the mechanism involved in chromosomal rearrangements in mammalian cells.

Furthermore, it has been suggested that some classes of repetitive sequences may contribute to gene regulation. In chicken, the CR1 sequence, which share features with retrovirus LTRs (Stumph et al., 1984), is supposed to change the chromosome structure and thus change gene expression (Scott et al., 1987). In addition, it has been shown that CR1 can function as a silencer element leading to the reduction of transcription (Baniahmad et al., 1987). In vertebrate, repetitive elements of the $\mathrm{Ll}$ and Alu sequences have also been shown to function as silencers (Laimans et al., 1986; Saffer and Thurston, 1989). p1 sequence has three repetitive sequences, all of which may contribute to gene regulation. Although 
further study is needed to substantiate this possibility, this clone may be useful to study not only chromosome organization but also the involvement of repetitive sequences in gene regulation.

Acknowledgment This work was supported by the Grant-in-Aid for Science Research on Priority Areas No. 03224104 from the Ministry of Education, Science and Culture of Japan and NIH grant HD 17449 to Dr. D. Patterson. We appreciate the support of Shin-Etu-Chemicals for these studies. The nucleotide sequence data reported will appear in GenBank under the accession number L05173.

\section{REFERENCES}

Baniahmad A, Muller M, Steiner C, Renkawitz R (1987): Activity of two different silencer elements of the chicken lysozyme gene can be compensated by enhancer elements. EMBO J 6: 2297-2303

Daniels GR, Deininger PL (1985): Integration site preferences of the Alu family and similar repetitive DNA sequences. Nucleic Acids Res 13: 8939-8954

Drabkin HA, Diaz M, Bradley CM, LeBeau MM, Rowley JD, Patterson D (1985): Isolation and analysis of the $21 \mathrm{q}^{+}$chromosome in the acute myelogenous leukemia $8 ; 21$ translocation: Evidence that c-mos is not translocated. Proc Natl Acad Sci USA 82: 464-468

Gardiner K, Aissani B, Bernardi G (1990a): A compositional map of human chromosome 21. EMBO J 9: $1853-1858$

Gardiner K, Horisberger M, Kraus J, Tantravahi U, Korenberg J, Rao V, Reddy S, Patterson D (1990b): Analysis of human chromosome 21: Correlation of physical and cytogenetic maps; gene and $\mathrm{CpG}$ island distribution. EMBO J 9: 25-34

Henikoff $S$ (1984): Unidirectional digestion with exonuclease III creates targeted breakpoints for DNA sequencing. Gene 28: 351-359

Henthorn PS, Mager DL, Huisman TH, Smithies O (1986): A gene deletion ending within a complex array of repeated sequences $3^{\prime}$ to the human $\beta$-globin gene cluster. Proc Natl Acad Sci USA 83: $5194-5198$

Jagadeeswaran P, Tuan D, Forget BG, Weissman SM (1982): A gene deletion ending at the midpoint of a repetitive DNA sequence in one form of hereditary persistence of fetal haemoglobin. Nature 296: 469-470

Jelinek WR, Schmid CW (1982): Repetitive sequences in eukaryotic DNA and their expression. Annu Rev Biochem 51: 813-844

Jurka J, Smith T (1988): A fundamental division in the Alu family of repeated sequences. Proc Natl Acad Sci USA 85: 4775-4778

Korenberg JR, Rykowski MC (1988): Human genome organization: Alu, lines, and the molecular structure of metaphase chromosome bands. Cell 53: 391-400

Kozak CA, Lawrence JB, Ruddle FH (1977): A sequential staining technique for the chromosomal analysis of the interspecific mouse/hamster and mouse/human somatic cell hybrids. Exp Cell Res 105: 109-117

Laimins L, Holmgren-Konig M, Khoury G (1986): Transcriptional "silencer" element in rat repetitive sequences associated with the rat insulin 1 gene locus. Proc Natl Acad Sci USA 83: 3151-3155

Lehrman MA, Russell DW, Goldstein JL, Brown MS (1986): Exon-Alu recombination deletes 5 kilobases from the low density lipoprotein receptor gene, producing a null phenotype in familial hypercholesterolemia. Proc Natl Acad Sci USA 83 : 3679-3683

Levy E, Carman MD, Fernandez-Madrid IJ, Power MD, Lieberburg I, Van Duinen SG, Bots GT, Luyendijk W, Frangione B (1990): Mutation of the Alzheimer's disease amyloid gene in hereditary cerebral hemorrhage, Duch type. Science 248: 1124-1126 
Mager DL, Henthorn PS (1984): Identification of a retrovirus-like repetitive element in human DNA. Proc Natl Acad Sci USA 81: 7510-7514

Mager DL, Henthorn PS, Smithies $O(1985)$ : A Chinese $G \gamma^{+}(A \gamma \delta \beta) 0$ thalassemia deletion: comparison to other deletions in the buman $\beta$-globin gene cluster and sequence analysis of the breakpoints. Nucleic Acids Res 13: 6559-6575

Moyzis RK, Torney DC, Meyne J, Buckingham JM, Wu JR, Burks C, Sirotkin KM, Goad WB (1989): The distribution of interspersed repetitive DNA sequences in the human genome. Genomics 4: 273-289

Oates DC, Patterson D (1977): Biochemical genetics of Chinese hamster cell mutants with deviant purine metabolism: characterization of Chinese hamster cell mutants defective in phosphoribosylpyrophosphate amidotransferase and phosphoribosylglycinamide synthetase and an examination of alternatives to the first step of purine biosynthesis. Somatic Cell Genet 3: 561577

Patterson D, Jones C, Morse H, Rumsby P, Miller Y, Davis R (1983): Structural gene coding for multifunctional protein carrying orotate phosphoribosyltransferase and OMP decarboxylase activity is located on long arm of human chromosome 3. Somatic Cell Genet 9: 359-374

Paulson KE, Deka N, Schmid CW, Misra R, Schindler CW, Ruch MG, Kadyk L, Leinwand L (1985): A transposon-like element in human DNA. Nature 316: 359-361

Petersen MB, Slaugenhaupt SA, Lewis JG, Warren AC, Chakravarti A, Antonarakis SE (1991): A genetic linkage map of 27 markers on human chromosome 21. Genomics 9: 407-419

Rubin CM, Houck CM, Deininger PL, Friedmann T, Schmid CW (1980): Partial nucleotide sequence of the 300-nucleotide interspersed repeated human DNA sequences. Nature 284: 372374

Saffer JD, Thurston SJ (1989): A negative regulatory element with properties similar to those of enhancers is contained within an Alu sequence. Mol Cell Biol 9: 355-364

Saiki RK, Gelfand DH, Stoffel S, Scharf SJ, Higuchi R, Horn GT, Mullis KB, Erlich HA (1988): Primer-directed enzymatic amplification of DNA with a thermostable DNA polymerase. Science 239: $487-491$

Scott MJ, Tsai MJ, O'Malley BW (1987): Deoxyribonuclease I sensitivity of the ovomucoid-ovoinhibitor gene complex in oviduct nuclei and relative location of CR1 repetitive sequences. Biochemistry 26: 6831-6840

Singer MF, Skowronski J (1985): Making sense out of LINES: Long interspersed repeated sequences in mammalian genomes. TLBS 10: 119-122

Skowronski J, Fanning TG, Singer MF (1988): Unit-length line-1 transcripts in human teratocarcinoma cells. Mol Cell Biol 8: 1385-1397

Steele PE, Rabson AB, Bryan T, Martin MA (1984): Distinctive termini characterize two families of human endogenous retroviral sequences. Science 225: 943-947

Stumph WE, Hodgson CP, Tsai MJ, O'Malley BW (1984): Genomic structure and possible retroviral origin of the chicken CR1 repetitive DNA sequence family. Proc Natl Acad Sci USA 81: $6667-6671$

Sun L, Paulson KE, Schmid CW, Kadyk L, Leinwand L (1984): Non-Alu family interspersed repeats in human DNA and their transcriptional activity. Nucleic Acids Res 12: 2669-2690

Van Keuren ML, Watkins PC, Drabkin HA, Jabs EW, Gusella JF, Patterson D (1986): Regional localization of DNA sequences on chromosome 21 using somatic cell hybrids. Am J Hum Genet 38: 793-804

Varmus HE (1982): Form and function of retroviral proviruses. Science 216: 812-820

Wallace MR, Andersen LB, Saulino AM, Gregory PE, Glover TW, Collins FS (1991): A de novo Alu insertion in neurofibromatosis type 1. Nature 353: 864-866

Weiner AM, Deininger PL, Efstratiadis A (1986): Nonviral retroposones: genes, pseudogenes, and transposable elements generated by the reverse flow of genetic information. Annu Rev Biochem 55: $631-661$ 
Wong C, Kazazian HH Jr, Stetten G, Earnshaw WC, Van Keuren ML, Antonarakis SE (1989) Molecular mechanism in the formation of a human ring chromosome 21. Proc Natl Acad Sci USA 86: 1914-1918

Zsebo KM, Williams DA, Geissler EN, Broudy VC, Martin FH, Atkins HL, Hsu RY, Birkett NC, Okino KH, Murdock. DC, Jaconsen FW, Langley KE, Smith KA, Takeishi T, Cattanach BM, Gall SJ, Suggs SV (1990): Stem cell factor is encoded at the SI locus of the mouse and is the ligand for the c-kit tyrosine kinase receptor. Cell 63: 213-224

Vol. 38, No. 3, 1993 